\title{
Tendencias de investigación sobre comunicación organizacional: la autoría española en revistas indexadas (2014-2018)
}

\author{
Organizational communication research \\ trends: Contributions by Spanish authors \\ in indexed journals (2014-2018)
}

\author{
María-Isabel Míguez-González; Carmen Costa-Sánchez
}

Cómo citar este artículo:

Míguez-González, María-Isabel; Costa-Sánchez, Carmen (2019). “Tendencias de investigación sobre comunicación organizacional: la autoría española en revistas indexadas (2014-2018)". El profesional de la información, v. 28, n. 5, e280510.

https://doi.org/10.3145/epi.2019.sep.10

Artículo recibido el 08-04-2019

Aceptación definitiva: 16-08-2019

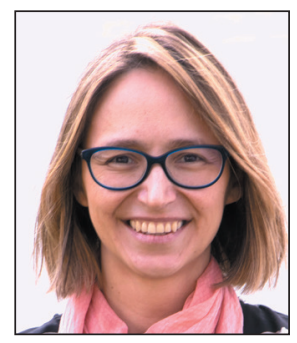

María-Isabel Míguez-González
http://orcid.org/0000-0002-0580-8493
Universidade de Vigo $\square$
Facultade de Ciencias Sociais e da Comunicación
Departamento de Comunicación Audiovisual e
Publicidade
Campus A Xunqueira, s/n.
36005 Pontevedra, España
mabelm@uvigo.es

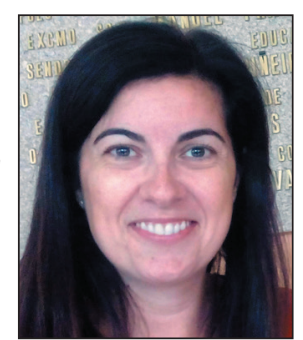

Carmen Costa-Sánchez

https://orcid.org/0000-0001-8154-9537

Universidade da Coruña

Facultade de Ciencias da Comunicación

Campus de Elviña, $\mathrm{s} / \mathrm{n}$.

15001 A Coruña, España

carmen.costa@udc.es

\section{Resumen}

Se ofrece una visión general de las tendencias de investigación sobre comunicación organizacional generada por autores españoles en revistas de alto impacto entre los años 2014 y 2018, atendiendo a aspectos como el año de publicación, el soporte o el número de citas obtenidas, así como las características de autoría y la temática abordada. Los resultados muestran una presencia discreta pero estable de este tipo de estudios, con variedad de soportes, sobre todo nacionales, y temáticas, aunque la comunicación online protagoniza casi un tercio de los artículos. La diversidad de autores y universidades también es notoria, pero un grupo reducido de instituciones concentra una parte importante de la producción científica. Destaca el elevado número de trabajos firmados en coautoría y un porcentaje de autoría femenina superior al $50 \%$. Más de la mitad de los artículos de la muestra nunca han sido citados.

\section{Palabras clave}

Comunicación organizacional; Comunicación corporativa; Relaciones públicas; Investigación; Comunicación académica; Revistas científicas; Bibliometría; Autoría; Tendencias; Scopus.

\begin{abstract}
An overview of the research trends on organizational communication generated by Spanish authors in high impact journals between 2014 and 2018 is offered. Variables such as the year of publication, the source or the number of citations, as well as characteristics of authorship and issues addressed are analyzed. The results show a discrete but stable presence of this type of studies, with variety of sources, especially Spanish journals, and issues, although online communication accounts for almost a third of total articles. The diversity of authors and universities is also notorious, but a small group of institutions concentrates an important part of the research. It is remarkable the high number of works signed in co-authorship, and a percentage of female authorship over $50 \%$. More than half of the items in the sample have never been cited.
\end{abstract}

\section{Keywords}

Organizational communication; Corporate communication; Public relations; Research; Scholarly communication; Academic journals; Bibliometrics; Authorship; Trends; Scopus. 


\section{Introducción}

El análisis de las tendencias de investigación en un determinado tema o en una disciplina concreta es esencial para valorar su evolución a lo largo del tiempo, detectar carencias, tanto desde el punto de vista de los contenidos investigados como de las metodologías, y medir la repercusión en la comunidad académica.

La utilidad de los estudios bibliométricos para describir las tendencias de investigación y actividad científica en comunicación ha sido destacada por numerosos autores (Borgman; Furner, 2002; Bauer; Bakkalbasi, 2005; Feeley, 2008). En el caso español, si bien desde principios del siglo XXI se produce la eclosión de los estudios sobre la investigación de la comunicación en España (Jones et al., 2000; Martínez-Nicolás, 2001; 2009; Castillo-Esparcia; Xifra, 2006; Xifra; Castillo-Esparcia, 2006; Soriano-Clemente, 2008), el incremento del peso de los artículos científicos en revistas de alto impacto para la consecución de acreditaciones y sexenios ha consolidado en los últimos años el desarrollo de este tipo de estudios, que ayudan a los investigadores a detectar los mejores canales para hacer públicos sus resultados de investigación (Míguez-González; Corbacho-Valencia; Baamonde-Silva, 2016).

A principios de la década podemos localizar varios trabajos que analizan la investigación publicada en revistas españolas de comunicación (Castillo-Esparcia; Carretón, 2010; Fernández-Quijada, 2010; 2011; Roca-Correa; Pueyo-Ayhan, 2012; Baladrón-Pazos; Correyero-Ruiz, 2012), profundizando en aspectos como el volumen de artículos publicados y su procedencia, las características de autoría, redes de colaboración de autores y universidades, metodologías, patrones de citación o las carencias de las propias revistas.

Las publicaciones internacionales también han despertado el interés de los investigadores, que necesitan cada vez más ampliar el rango de revistas de alto impacto a las que optar para poder cumplir los requisitos exigidos por Aneca y Cneai. En este sentido resultan de utilidad estudios como los de:

- Castillo-Esparcia, Rubio-Moraga y Almansa-Martínez (2012), que analizan las diez publicaciones de comunicación con mayor impacto del Social Sciences Citation Index;

- Méndez-Rátiva y Gregorio-Chaviano (2014), que obtienen indicadores de productividad de autores, instituciones y países, e indicadores de citación a partir del análisis de cinco publicaciones internacionales indexadas en WoS;

- Delgado-López-Cózar y Repiso (2013), que comparan las bases de datos que se emplean para la medición de impacto, aplicadas a las revistas de comunicación.

Costa-Sánchez (2017) sintetiza varias tendencias apuntadas en los estudios previos en torno a esta cuestión:

- predominio de la producción individual o en pareja, debido a que la autoría múltiple (más de tres autores) es penalizada en los sistemas de acreditación;

- selección de revistas en función de los beneficios en términos curriculares, lo que prima la voluntad de publicación en revistas de alto impacto;

- escasa visibilidad de la investigación española en comunicación en las revistas internacionales;

- bajas tasas de citación;

- predominio de algunas líneas de investigación sobre otras.

También son abundantes los estudios que se centran en disciplinas específicas dentro de la comunicación, como el periodismo (Martínez-Nicolás; Saperas; Carrasco-Campos, 2017), publicidad (Baladrón-Pazos; Correyero-Ruiz; Manchado-Pérez, 2014), relaciones públicas (Míguez, 2011; Míguez-González; Baamonde-Silva; Corbacho-Valencia, 2014; Míguez-González; Corbacho-Valencia; Baamonde-Silva, 2016) o en aspectos concretos como las marcas territorio y la diplomacia pública (Compte-Pujol; De-Urquijo; Matilla, 2016) o la narrativa transmedia (Vicente-Torrico, 2017).

Sin embargo, los análisis específicos sobre comunicación organizacional, como el de Pazmiño-Santacruz y González-Alonso (2014), son escasos y de carácter exploratorio. Este estudio pretende cubrir esa laguna, aportando una visión general de las tendencias en la investigación sobre comunicación organizacional generada por autores españoles en revistas de alto impacto, determinando las temáticas más estudiadas y describiendo las características de autoría de estas publicaciones. Para ello se comienza aclarando el concepto de comunicación organizacional.

\section{Comunicación organizacional: cuestiones terminológicas}

Según Jablin y Putnam (2000), a finales de los años treinta o inicios de los cuarenta nació en Estados Unidos una disciplina denominada organizational communication. Según Putnam este concepto partía de cuatro tradiciones:

- speech communication,

- persuasión,

- discurso público, y

- teoría de la comunicación humana,

y se identifica con las formas en que la comunicación puede contribuir a la efectividad de la empresa (Putnam, 2002). Este enfoque, que se consolidó en los años 60 , se centra en las relaciones informativas que se producen dentro de la empresa y por tanto se orienta hacia la gestión de la comunicación interna. Desde esta perspectiva, la comunicación organizacional se ha definido como el "flujo de mensajes dentro de una red de relaciones interdependientes" o como el 
"proceso mediante el cual un individuo, o una de las subpartes de la organización, se pone en contacto con otro individuo u otra subparte" (León-Duarte, 2006, p. 292).

Esta tradición continúa teniendo hoy un desarrollo relevante en estudios de recursos humanos.

Sin embargo, en comunicación el uso del concepto se ha extendido más allá de su significado original y el término convive con otros en un contexto de importante confusión terminológica (Míguez-González, 2015), adquiriendo diferentes matices en función de los autores y de los lugares geográficos donde se emplea.

Varios autores del contexto académico español utilizan la expresión comunicación organizacional para hacer referencia, de un modo genérico, a la gestión integral de la comunicación en las organizaciones (Losada-Díaz, 2004; García-Orosa, 2006; Timoteo-Álvarez, 2013). Entendido de este modo, según García-Orosa (2006), el término se emplea tanto para definir la comunicación realizada por las instituciones o comunicación institucional, como la de las empresas (comunicación empresarial), si bien su utilización debería extenderse también a la comunicación ejecutada desde las organizaciones del tercer sector.

Este modo de comprender el concepto remite a otro interrogante: ¿qué se entiende por comunicación de las organizaciones o qué elementos se incluyen dentro de ésta? Torres-Alonso y Alejo-Becerra plantean que el nacimiento de la comunicación organizacional obedece a la posibilidad de

"cohesionar disciplinas que hasta el momento de su surgimiento funcionaban de manera inconexa; tales como la publicidad, las relaciones públicas o el marketing" (Torres-Alonso; Alejo-Becerra, 2011).

En esta línea, León-Duarte (2006) menciona la tendencia de grandes organizaciones de tener una única área de gestión de comunicación organizacional que aúna comunicación interna, externa y comercial y Noguera-Vivo afirma que

"los enfoques modernos de comunicación organizacional se orientan ahora desde una perspectiva multidisciplinar y que se sustenta desde tres pilares: la comunicación interna, la corporativa y la de marketing" (Noguera-Vivo, 2005, p. 363).

Sin embargo, el concepto de comunicación corporativa que menciona Noguera-Vivo como parte de la comunicación organizacional también se asocia con la idea de una gestión integrada de los tipos de comunicación. Así, según Van-Riel (1997), autor de referencia, la comunicación corporativa incluiría la comunicación de dirección o management communication, la comunicación de marketing y la comunicación organizativa. Y para Costa (2011), la comunicación corporativa estaría integrada por la comunicación institucional o referida a la presidencia, la comunicación mercadológica y la comunicación organizacional, entendida como comunicación interna.

Introduciendo el factor geográfico, cabe apuntar que lo que Van-Riel denomina corporate communication y que en España ha sido traducido como comunicación corporativa (Van-Riel, 1997), en otros casos, sobre todo en Latinoamérica, se ha traducido como comunicación organizacional (Van-Riel, 2003; Goldhaber, 2003; Valle, 2003; Guzmán, 2012; De-Castro, 2015), determinando claramente la sinonimia entre ambos términos.

Otro término en conflicto dentro de este panorama terminológico es el de relaciones públicas, una disciplina histórica cuyas actividades se integran, según los teóricos de la comunicación corporativa, dentro de ésta (Van-Riel; Fombrun, 2007; Costa, 2011; Villafañe, 1993; Cornelissen, 2014). Sin embargo, en relaciones públicas se asume por lo general que ambos términos son sinónimos (Arceo-Vacas, 2005; Xifra, 2005) y que la preferencia por la utilización del concepto comunicación corporativa en el ámbito empresarial se debe a una concepción errónea de lo que son las relaciones públicas (Wilcox; Cameron; Xifra, 2012). Autores como Dilenschneider (2000) o Christesen y Cornelissen (2010), desde una posición intermedia, plantean que la comunicación corporativa puede entenderse como una versión más amplia o desarrollada de la tradicional disciplina de las relaciones públicas.

En cualquier caso, dado que las relaciones públicas se aproximan conceptualmente a la comunicación corporativa y ésta a la comunicación organizacional, es lógico que el término comunicación organizacional se emplee también como sinónimo de relaciones públicas, como sucede en la compilación de Castillo-Esparcia (2005), cuyos autores proceden en su mayoría de la disciplina definida por Bernays.

Saladrigas-Medina sintetiza así esta situación terminológica de la comunicación organizacional:

"Como actividad ha recibido múltiples denominaciones. Entre las más reconocidas, las que la sitúan solamente dentro de la organización -comunicación organizacional, comunicación interna y comunicación institucional-; las que la ubican hacia fuera de la organización -comunicación externa y relaciones públicas-y las que integran ambos ámbitos la denominan comunicación integrada, global, corporativa y también institucional" (Saladrigas-Medina, 2005). 
Cabría mencionar además que la utilización del concepto dirección de comunicación o communication management también se asocia obviamente con la gestión de la comunicación en las organizaciones, ya sea desde la perspectiva de las relaciones públicas (Almansa, 2006; Mut-Camacho, 2006; Morales; Enrique, 2007; Gutiérrez-García; Rodríguez-Salcedo, 2009) o desde la perspectiva de la comunicación corporativa (Benavides et al., 2001), por lo que presenta una relación directa con la noción de comunicación organizacional que manejamos en este texto.

Por último, podría matizarse que si bien la comunicación de marketing entra dentro de la gestión de la comunicación corporativa u organizacional según diferentes autores ya mencionados, el papel que desarrolla el director de comunicación, como cabeza visible de los departamentos de comunicación de las organizaciones, se basa en la coordinación de las diferentes formas de comunicación de la entidad pero se centra de forma patente en la comunicación no comercial (Dircom, 2013), quedando la gestión específica de la comunicación comercial reservada a los departamentos de marketing. Por este motivo, a efectos de este estudio entenderemos por investigaciones de comunicación organizacional las que tienen que ver con la comunicación de cualquier tipo de organización (empresas, instituciones y tercer sector) desde una perspectiva no marketiniana.

\section{Objetivos y método}

El estudio se llevó a cabo sobre el total de artículos sobre comunicación organizacional publicados por autores españoles en revistas indexadas en Scopus en el quinquenio 2014-2018 y recogidos en esta base de datos a 20 de enero de 2019. Dado que la búsqueda se realizó la última semana de enero de 2019, ha de considerarse que los artículos presentes en números de revista correspondientes a 2018 que aún no hubiesen sido incorporados al directorio de Scopus en la fecha de recogida de la información no aparecerán representados en la muestra.

En el proceso de búsqueda se procedió a la localización de artículos que contuviesen en su título, abstract o palabras clave los términos, tanto en castellano como en inglés, que, de acuerdo con el planteamiento teórico, podrían devolver en los resultados artículos relativos, en un sentido amplio, a la comunicación organizacional (tabla 1). Para cada búsqueda los artículos se filtraron por país (España).

Del total de artículos localizados, en un primer proceso de revisión se eliminaron las duplicaciones por cada par de términos (artículos que aparecían en la búsqueda del término en castellano y que ya habían aparecido en la búsqueda del término en inglés), así como algunos casos de artículos que dentro de la búsqueda de un mismo término y un mismo idioma aparecían repetidos y algún texto que no contaba con ningún autor español o perteneciente a alguna universidad española.

Una vez obtenida esta primera base de datos de 315 artículos válidos se realizó manualmente una revisión en la que se eliminaron los artículos que a pesar de cumplir los criterios de búsqueda, no encajaban dentro de los parámetros establecidos para la investigación: Se eliminaron de la muestra los artículos que aluden a la comunicación organizacional o similares pero que:

- centran la investigación en disciplinas ajenas a la comunicación (lingüística, tecnología, informática, empresa...);

- se centran claramente en otros aspectos de la comunicación que no encajan dentro de la investigación en comunicación organizacional (publicidad, comunicación audiovisual, medios de comunicación de masas, periodismo...);

- se centran en aspectos didácticos o de metodología e innovación docente.

Este proceso de revisión fue realizado por dos investigadores de forma independiente. El nivel de desacuerdo fue inferior a un $5 \%$ de los casos y se resolvió mediante una discusión que condujo a una decisión consensuada sobre los casos dudosos, para generar un listado de 154 artículos incluidos. Finalmente se unificaron las búsquedas por términos y se eliminaron los artículos duplicados, resultando una muestra final de 128 artículos.

Tabla 1. Construcción de la muestra

\begin{tabular}{|c|c|c|c|c|}
\hline Término de búsqueda & $\begin{array}{c}\text { Artículos recu- } \\
\text { perados }\end{array}$ & $\begin{array}{l}\text { Artículos } \\
\text { válidos }\end{array}$ & $\begin{array}{l}\text { Artículos } \\
\text { excluidos }\end{array}$ & $\begin{array}{l}\text { Artículos } \\
\text { incluidos }\end{array}$ \\
\hline Organizational communication + comunicación organizacional & $24+2$ & 24 & 12 & 12 \\
\hline Corporate communication + comunicación corporativa & $45+8$ & 46 & 8 & 38 \\
\hline Institutional communication + comunicación institucional & $18+1$ & 17 & 4 & 13 \\
\hline Business communication + comunicación empresarial & $21+2$ & 21 & 12 & 9 \\
\hline Communication management + dirección de comunicación & $34+2$ & 35 & 18 & 17 \\
\hline Public relations + relaciones públicas & $179+20$ & 172 & 107 & 65 \\
\hline Totales & 356 & 315 & 161 & 154 \\
\hline \multicolumn{4}{|c|}{ Artículos duplicados entre diferentes bloques de términos de búsqueda } & 26 \\
\hline \multicolumn{4}{|l|}{ Total de artículos de la muestra } & 128 \\
\hline
\end{tabular}


Sobre estos 128 artículos se aplicó una plantilla de análisis basada en los elementos estudiados en investigaciones previas (Castillo-Esparcia; Carretón, 2010; Míguez-González; Baamonde-Silva; Corbacho-Valencia, 2014; Míguez-González; Corbacho-Valencia; Baamonde-Silva, 2016). En la plantilla se contemplaron las siguientes variables objetivas:

- término o términos de búsqueda que permitieron localizar el artículo,

- año de publicación,

- revista,

- número de citas,

- número de autores y nombre,

- filiación y sexo de cada uno.

Además se añadieron dos variables para la clasificación de los artículos:

- tema abordado y

- tipo de organización sobre la que trata.

Por lo que respecta al análisis de contenido, para la categorización de los artículos según el tema y el tipo de organización se aplicó una estrategia mixta (Andréu-Abela, 2001). Se combinó una codificación deductiva por parte de uno de los investigadores, tomando como referencia la clasificación propuesta por Míguez-González, Baamonde-Silva y Corbacho-Valencia (2014) para el ámbito de las relaciones públicas, con una codificación inductiva por parte del otro investigador. La categorización final resultante se generó por consenso de los dos investigadores, adoptando alguna de las categorías del estudio previo y añadiendo o modificando otras en función de los resultados de la codificación inductiva.

\section{Resultados}

\subsection{Características de los artículos}

La muestra se compone de 128 artículos publicados entre 2014 y 2018. Desde el punto de vista temporal, se aprecia bastante estabilidad en el número de artículos publicados por año, con un ligero pico de producción en los años 2016 y 2017 (tabla 2). Cabe matizar que estos datos están afectados por la publicación de números monográficos o especiales dedicados a comunicación organizacional, como los publicados por la revista Prisma social en 2015 y 2018 o la revista Opción en 2016, lo que podría explicar el pico en ese año en concreto. De todos modos, debido a los criterios de búsqueda especificados, no todos los artículos de dichos monográficos

"Dirección de comunicación" o communication management también se asocia obviamente con la gestión de la comunicación en las organizaciones, ya sea desde la perspectiva de las relaciones públicas o desde la perspectiva de la comunicación corporativa, por lo que presenta una relación directa con la noción de "comunicación organizacional" se encuentran en la muestra, ya que en algunos casos su temática presentaba una vinculación mayor con otros temas (marketing, empresa, publicidad, comunicación audiovisual...) que con la comunicación organizacional tal y como se ha definido anteriormente.

El índice de cooperación, es decir, la ratio entre número de firmas (incluyendo autores duplicados) y artículos publicados es de 2,21. Una cuarta parte de los artículos están firmados por un único autor, pero lo más habitual es encontrar trabajos firmados por dos o tres autores (32\% y 39\% respectivamente). En los años 2017 y 2018 se registra una reducción del porcentaje de artículos firmados por un único autor y un incremento de los firmados por tres autores (tabla 2).

Los 128 trabajos suman 171 citas (promedio de 1,34 citas por artículo, con una desviación estándar de 2,96), aunque 27 se corresponden con un único artículo (el más citado). El 58\% de los textos no han recibido ninguna cita y sólo un $4 \%$ acumulan seis o más citas (tabla 2). No se observa correlación entre el número de citas y el número de autores firmantes de los artículos $(r=0,115)$. Sí hay una relación significativa $(p<0,01)$, como es de esperar, entre el año de publicación y el rango de citas obtenidas por los artículos publicados, con una tendencia a la acumulación de citas conforme pasa el tiempo.

En cuanto a la temática, un 38\% de los artículos ponen su foco en la comunicación online. La gestión y estrategia de comunicación es el siguiente tema de interés (14\%), seguido del profesionalismo (9\%). No se observan variaciones significativas en la temática en función de los años; entre 2014 y 2017 se detecta un incremento anual importante del número de artículos relacionados con la comunicación online, pero este tema sufre una caída importante en 2018 que rompe con la tendencia ascendente (tabla 2). Aunque para algunos temas se detecta un promedio de citas más elevado (campañas y comunicación online y móvil) y la correlación entre número de artículos por temática y número de citas es muy elevada $(r=0,9)$, dado el reducido número de artículos por temática no resulta posible generalizar este resultado.

Con respecto al sector al que se refiere el estudio, un $31,25 \%$ se centran en el empresarial y un $20,31 \%$ no se refieren a ningún sector o tipo de organización en concreto; estos últimos son los que obtienen un promedio de citas más elevado (tabla 1). La correlación entre el número de artículos por sector y el número de citas es elevada $(r=0,89)$. 
Tabla 2. Características de los artículos por año de publicación

\begin{tabular}{|c|c|c|c|c|c|c|c|}
\hline \multirow[b]{2}{*}{ Número de autores } & \multicolumn{6}{|c|}{ Número de artículos publicados por año } & \multirow[t]{2}{*}{$\begin{array}{l}\text { Promedio } \\
\text { citas/art }\end{array}$} \\
\hline & 2014 & 2015 & 2016 & 2017 & 2018 & Total (\%) & \\
\hline 1 & 3 & 5 & 15 & 7 & 3 & $33(25,78)$ & 1,06 \\
\hline 2 & 7 & 12 & 6 & 9 & 7 & $41(32,03)$ & 1,29 \\
\hline 3 & 10 & 7 & 9 & 13 & 11 & $50(39,06)$ & 1,12 \\
\hline 4 & 0 & 1 & 0 & 1 & 0 & $2(1,56)$ & 13,50 \\
\hline 5 & 0 & 1 & 0 & 0 & 1 & $2(1,56)$ & 0,00 \\
\hline $\begin{array}{l}\text { Total } \\
(\%)\end{array}$ & $\begin{array}{c}20 \\
(15,63)\end{array}$ & $\begin{array}{c}26 \\
(20,31)\end{array}$ & $\begin{array}{c}30 \\
(23,44)\end{array}$ & $\begin{array}{c}30 \\
(23,44)\end{array}$ & $\begin{array}{c}22 \\
(17,19)\end{array}$ & & 1,34 \\
\hline Citas recibidas** & 2014 & 2015 & 2016 & 2017 & 2018 & Total (\%) & \\
\hline 0 citas & 5 & 15 & 16 & 17 & 21 & $74(57,81)$ & \\
\hline $1-2$ citas & 6 & 6 & 8 & 10 & 1 & $31(24,22)$ & \\
\hline 3-5 citas & 6 & 3 & 6 & 3 & 0 & $18(14,06)$ & \\
\hline 6-10 citas & 3 & 1 & 0 & 0 & 0 & $4(3,13)$ & \\
\hline Más de 10 & 0 & 1 & 0 & 0 & 0 & $1(0,78)$ & \\
\hline \multicolumn{8}{|l|}{${ }^{* *} \mathrm{p}<0,01$} \\
\hline Tema & 2014 & 2015 & 2016 & 2017 & 2018 & Total (\%) & \\
\hline Campañas & 2 & 0 & 1 & 1 & 0 & $4(3,13)$ & 3,75 \\
\hline Comunicación audiovisual & 1 & 0 & 1 & 2 & 0 & $4(3,13)$ & 1,00 \\
\hline Comunicación corporativa & 1 & 2 & 3 & 1 & 0 & $7(5,47)$ & 1,14 \\
\hline Comunicación de crisis & 2 & 0 & 0 & 0 & 0 & $2(1,56)$ & 2,00 \\
\hline Comunicación interna & 1 & 1 & 1 & 0 & 1 & $4(3,139$ & 0,75 \\
\hline Comunicación online y móvil & 5 & 7 & 11 & 17 & 9 & $49(38,28)$ & 2,02 \\
\hline Eventos y protocolo & 0 & 1 & 0 & 1 & 1 & $3(2,34)$ & 0,00 \\
\hline Gestión y estrategia comunicativa & 3 & 5 & 4 & 3 & 3 & $18(14,06)$ & 0,33 \\
\hline Metainvestigación & 1 & 2 & 2 & 0 & 3 & $8(6,25)$ & 0,88 \\
\hline Opinión pública & 1 & 0 & 0 & 1 & 0 & $2(1,56)$ & 0,00 \\
\hline Profesionalismo & 1 & 4 & 2 & 3 & 2 & $12(9,38)$ & 1,33 \\
\hline Relaciones con los medios y publicity & 2 & 1 & 1 & 1 & 2 & $7(5,47)$ & 0,00 \\
\hline RSC & 0 & 2 & 3 & 0 & 1 & $6(4,69)$ & 0,50 \\
\hline Teoría básica & 0 & 1 & 1 & 0 & 0 & $2(1,56)$ & 3,00 \\
\hline Sector de actividad & 2014 & 2015 & 2016 & 2017 & 2018 & Total (\%) & \\
\hline Agencias y consultoras de comunicación & 0 & 1 & 1 & 0 & 0 & $2(1,56)$ & 1,00 \\
\hline Deportes & 0 & 3 & 2 & 0 & 1 & $6(4,69)$ & 0,00 \\
\hline Política & 0 & 1 & 2 & 1 & 2 & $6(4,69)$ & 1,17 \\
\hline Empresas en general & 9 & 9 & 6 & 10 & 6 & $40(31,25)$ & 1,18 \\
\hline Entidades sin ánimo de lucro & 1 & 1 & 3 & 2 & 1 & $8(6,25)$ & 0,63 \\
\hline General & 3 & 5 & 7 & 4 & 7 & $26(20,31)$ & 2,38 \\
\hline Hospitales & 0 & 0 & 3 & 1 & 1 & $5(3,91)$ & 0,20 \\
\hline Instituciones públicas & 0 & 1 & 4 & 4 & 1 & $10(7,81)$ & 1,20 \\
\hline Museos & 1 & 1 & 1 & 4 & 2 & $9(7,03)$ & 2,00 \\
\hline Turismo & 3 & 3 & 0 & 3 & 0 & $9(7,03)$ & 1,10 \\
\hline Universidades & 3 & 1 & 1 & 1 & 1 & $7(5,47)$ & 1,00 \\
\hline
\end{tabular}

\subsection{Soportes}

Los trabajos se han publicado en 31 revistas. El 38,71\% de las revistas, publicadas en la Península Ibérica, aglutinan más del $57 \%$ de los artículos, mientras que algo menos del $19 \%$ se publican en revistas latinoamericanas y casi una cuarta parte en otras publicaciones internacionales; sin embargo, en estas últimas es donde se obtiene una ratio de citas por artículo más elevada.

Si desglosamos los datos por revista observamos que cuatro de ellas acumulan el $45 \%$ de los artículos publicados: $O p$ ción, El profesional de la información (EPI), Public relations review y Prisma social, mientras que diecisiete de las revistas 
están representadas en la muestra por un único artículo. Si atendemos a la ratio de citas por artículo, las 7 revistas que alcanzan o superan las 3 citas por artículo son internacionales. Communication and society es la única española que se aproxima a esta ratio $(2,89)$.

Tabla 3. Distribución de publicaciones y citas por revistas

\begin{tabular}{|c|c|c|c|c|c|c|}
\hline \multicolumn{3}{|l|}{ Revistas } & \multicolumn{2}{|c|}{ Artículos } & \multicolumn{2}{|c|}{ Citas } \\
\hline Tipo de revista & $\mathbf{n}$ & $\%$ & $\mathbf{n}$ & $\%$ & $\mathbf{n}$ & $\begin{array}{c}\text { Ratio citas/ } \\
\text { art. }\end{array}$ \\
\hline Españolas y portuguesas & 12 & 38,71 & 73 & 57,03 & 78 & 1,08 \\
\hline Latinoamericanas & 5 & 16,13 & 24 & 18,75 & 11 & 0,34 \\
\hline Otras internacionales & 14 & 45,16 & 31 & 24,22 & 80 & 3,33 \\
\hline \multicolumn{7}{|l|}{ Nombre de la revista } \\
\hline American behavioral scientist & & & 2 & 0,78 & 0 & 0,00 \\
\hline Athenea digital & & & 1 & 7,03 & 0 & 0,00 \\
\hline Business communication quarterly & & & 1 & 5,47 & 4 & 4,00 \\
\hline Catalan journal of communication and cultural studies & & & 9 & 11,72 & 9 & 1,00 \\
\hline Communication and society & & & 7 & 4,69 & 20 & 2,86 \\
\hline Communication research & & & 1 & 5,47 & 5 & 5,00 \\
\hline Corporate communications & & & 2 & 0,78 & 0 & 0,00 \\
\hline Corporate reputation review & & & 1 & 9,38 & 0 & 0,00 \\
\hline Cuadernos.info & & & 1 & 0,78 & 0 & 0,00 \\
\hline El profesional de la información (EPI) & & & 15 & 5,47 & 19 & 1,27 \\
\hline Espacios & & & 1 & 4,69 & 0 & 0,00 \\
\hline Estudios sobre el mensaje periodístico & & & 6 & 1,56 & 0 & 0,00 \\
\hline Historia y comunicación social & & & 7 & 0,78 & 9 & 1,29 \\
\hline ILU & & & 1 & 0,78 & 1 & 1,00 \\
\hline Intangible capital & & & 1 & 1,56 & 0 & 0,00 \\
\hline International journal of information management & & & 1 & 0,78 & 4 & 4,00 \\
\hline Journal of communication management & & & 3 & 0,78 & 10 & 3,33 \\
\hline Journal of fashion marketing and management & & & 1 & 0,78 & 3 & 3,00 \\
\hline Journal of promotion management & & & 1 & 2,34 & 4 & 4,00 \\
\hline Journal of public relations research & & & 1 & 0,78 & 2 & 2,00 \\
\hline Nationalities papers & & & 1 & 0,78 & 0 & 0,00 \\
\hline Observatorio & & & 6 & 0,78 & 3 & 0,50 \\
\hline Opción & & & 16 & 0,78 & 1 & 0,06 \\
\hline Palabra clave & & & 5 & 10,94 & 10 & 2,00 \\
\hline Prisma social & & & 12 & 0,78 & 3 & 0,25 \\
\hline Public relations review & & & 14 & 0,78 & 48 & 3,43 \\
\hline Revista española de documentación científica & & & 1 & 0,78 & 0 & 0,00 \\
\hline Revista latina de comunicación social & & & 7 & 0,78 & 14 & 2,00 \\
\hline Scientometrics & & & 1 & 12,50 & 0 & 0,00 \\
\hline Signo y pensamiento & & & 1 & 3,91 & 0 & 0,00 \\
\hline Tourism, culture and communication & & & 1 & 0,78 & 0 & 0,00 \\
\hline
\end{tabular}

La tabla de frecuencias entre revistas concretas y temas no arroja datos significativos. Sin embargo, se observa que el peso de algunos temas varía de forma importante en función del origen geográfico de las revistas. Si bien los artículos sobre comunicación online y móvil son los predominantes en todo tipo de revistas, su peso es ligeramente superior en el grupo de las españolas y portuguesas. Una cuarta parte de los trabajos publicados en revistas latinoamericanas tratan sobre gestión comunicativa, una temática a la que se presta una atención relevante también en las revistas españolas y portuguesas. En cambio, resulta casi inexistente en otras publicaciones internacionales, en las que el profesionalismo adquiere la segunda posición. Las revistas latinoamericanas son las que concentran un porcentaje mayor de textos sobre comunicación corporativa (tabla 4). 
Tabla 4. Distribución de artículos por temática y origen de las revistas

\begin{tabular}{|c|c|c|c|c|c|c|}
\hline & \multicolumn{2}{|c|}{ Españolas y portuguesas } & \multicolumn{2}{|c|}{ Latinoamericanas } & \multicolumn{2}{|c|}{ Otras internacionales } \\
\hline & $\mathbf{n}$ & $\% *$ & $\mathbf{n}$ & $\% *$ & $\mathbf{n}$ & $\% *$ \\
\hline Campañas & 2 & 2,78 & 0 & 0 & 2 & 6,25 \\
\hline Comunicación audiovisual & 3 & 4,17 & 1 & 4,17 & 0 & 0 \\
\hline Comunicación corporativa & 3 & 4,17 & 3 & 12,50 & 1 & 3,13 \\
\hline Comunicación de crisis & 1 & 1,39 & 0 & 0 & 1 & 3,13 \\
\hline Comunicación interna & 2 & 2,78 & 1 & 4,17 & 1 & 3,13 \\
\hline Comunicación online y móvil & 33 & 45,83 & 7 & 29,17 & 9 & 28,13 \\
\hline Eventos y protocolo & 0 & 0 & 2 & 8,33 & 1 & 3,13 \\
\hline Gestión & 11 & 15,28 & 6 & 25 & 1 & 3,13 \\
\hline Metainvestigación & 3 & 4,17 & 2 & 8,33 & 3 & 9,38 \\
\hline Opinión pública & 2 & 2,78 & 0 & 0 & 0 & 0 \\
\hline Profesionalismo & 5 & 6,94 & 1 & 4,17 & 6 & 18,75 \\
\hline Relaciones con los medios y publicity & 6 & 8,33 & 0 & 0 & 1 & 3,13 \\
\hline RSC & 2 & 2,78 & 1 & 4,17 & 3 & 9,38 \\
\hline Teoría básica & 0 & 0 & 0 & 0 & 2 & 6,25 \\
\hline
\end{tabular}

Tampoco resulta especialmente reveladora la tabla de frecuencias entre origen de las revistas y sectores de actividad, más allá de la constatación de que los artículos que más abundan en revistas internacionales (43,75\% de los publicados en ellas) son los que no abordan ningún sector específico.

\subsection{Autoría y filiación}

Por lo que respecta a la autoría, para los 128 trabajos publicados se registran 187 autores únicos, de los que un $54 \%$ son mujeres y un $46 \%$ son hombres. De los 33 artículos firmados por un único autor, el $55 \%$ están firmados por una mujer y el $45 \%$ por un hombre, con una proporción, por tanto, similar a la de presencia general de autoras y autores en la muestra. En los 95 textos con más de un autor, aparece una mujer como primera firmante en el $60 \%$ de los casos y un hombre en el $40 \%$. Un $21,9 \%$ de los artículos están firmados sólo por mujeres y un $28,1 \%$ sólo por hombres, pero en los trabajos firmados por más de un autor predominan los equipos mixtos (tabla 5).

De los 187 autores únicos, 139 firman solamente un artículo, lo que implica un 74,3\% de índice de transitoriedad, y 41 (22\%) firman dos o tres. Son muy pocos los autores por tanto que tienen presencia en cuatro o más trabajos (3,7\%) (tabla 5). Los tres autores con mayor presencia en la muestra (con nueve, ocho y siete artículos firmados) son mujeres.

Tabla 5. Distribución de artículos en función del sexo de sus autores

\begin{tabular}{|c|c|c|c|}
\hline No de autores & $\begin{array}{c}\text { Artículos firmados sólo por } \\
\text { mujeres }\end{array}$ & $\begin{array}{c}\text { Artículos firmados sólo por } \\
\text { hombres }\end{array}$ & $\begin{array}{c}\text { Artículos firmados por equipos } \\
\text { mixtos }\end{array}$ \\
\hline 1 & 18 & 15 & - \\
\hline 2 & 10 & 4 & 22 \\
\hline 3 & 8 & 0 & 38 \\
\hline 4 & 0 & 0 & 2 \\
\hline 5 & 0 & $21,9 \%$ & 2 \\
\hline \\
\hline muestra
\end{tabular}

En cuanto a las instituciones, en la muestra aparecen representadas 44 universidades españolas, además de 16 extranjeras y dos instituciones no universitarias. En la tabla 6 se presentan las doce universidades españolas que están presentes en un mayor número de artículos dentro de la muestra. Las restantes universidades están presentes en cuatro artículos o menos.

En cuanto al número de autores, la Universidad de Alicante, la Complutense y la de Málaga son las que más aportan, seguidas de la Rovira i Virgili y la Rey Juan Carlos. Entre las cinco, acumulan 67 autores, lo que supone un 35,83\% de los autores de la muestra. Once de las 44 universidades españolas están representadas por un único autor y trece de ellas, por dos. La Universidad Rey Juan Carlos es la que suma más firmas (tabla 6). 
Tabla 6. Doce universidades españolas con mayor presencia en la muestra

\begin{tabular}{|c|c|c|c|c|c|c|}
\hline \multirow[t]{2}{*}{ Universidad } & \multicolumn{2}{|c|}{$\begin{array}{l}\text { Artículos de la muestra en } \\
\text { los que tiene presencia }\end{array}$} & \multicolumn{2}{|c|}{$\begin{array}{c}\text { Autores que aporta a la } \\
\text { muestra (\% sobre el total de } \\
\text { autores) }\end{array}$} & \multirow{2}{*}{$\begin{array}{c}\text { Presencia } \\
\text { total (firmas } \\
\text { que suma)* } \\
\text { n }\end{array}$} & \multirow[t]{2}{*}{$\begin{array}{c}\text { Ratio } \\
\text { firmas / art }\end{array}$} \\
\hline & $\mathbf{n}$ & $\%$ & $\mathbf{n}$ & $\%$ & & \\
\hline Universidad Rey Juan Carlos & 14 & 10,94 & 11 & 5,88 & 27 & 1,93 \\
\hline Universitat Rovira i Virgili & 13 & 10,16 & 13 & 6,95 & 18 & 1,38 \\
\hline Universidad de Málaga & 12 & 9,38 & 14 & 7,49 & 20 & 1,67 \\
\hline Universidad Complutense de Madrid & 12 & 9,38 & 14 & 7,49 & 15 & 1,25 \\
\hline Universidad de Alicante & 10 & 7,81 & 15 & 8,02 & 21 & 2,10 \\
\hline Universidad de Cádiz & 10 & 7,81 & 7 & 3,74 & 13 & 1,30 \\
\hline Universidade da Coruña & 10 & 7,81 & 2 & 1,07 & 10 & 1,00 \\
\hline Universidad de Valladolid & 7 & 5,47 & 7 & 3,74 & 9 & 1,29 \\
\hline Blanquerna Universitat Ramon Llull & 7 & 5,47 & 5 & 2,67 & 11 & 1,57 \\
\hline Universitat de Vic & 7 & 5,47 & 4 & 2,14 & 7 & 1,00 \\
\hline Universidade de Vigo & 6 & 4,69 & 8 & 4,28 & 14 & 2,33 \\
\hline Universidad Católica de Murcia & 6 & 4,69 & 6 & 3,21 & 9 & 1,50 \\
\hline
\end{tabular}

\section{Discusión y conclusiones}

Aunque no existen investigaciones previas sobre el mismo objeto de estudio con las que se puedan establecer comparaciones exactas entre variables, sí es posible comparar algunos resultados con los datos aportados por otros estudios bibliométricos sobre comunicación en general y por estudios concretos sobre la investigación en relaciones públicas, dada la elevada coincidencia temática.

Desde este punto de vista, cabe señalar que la investigación española sobre comunicación organizacional tiene una presencia discreta pero estable en las revistas académicas indexadas en Scopus. Esta presencia se reparte en un elevado número de publicaciones y, aunque la variedad de revistas internacionales en las que se puede localizar algún artículo de autores españoles es elevada, la mayoría de la producción se concentra en las publicaciones nacionales. El profesional de la información es la revista española indexada en Scopus que más artículos sobre comunicación organizacional ha publicado en el último lustro.

En cuanto a la filiación de los autores, si bien varios estudios previos (Castillo-Esparcia; Carretón, 2010; Míguez-González; Baamonde-Silva; Corbacho-Valencia, 2014) aluden a la variedad de universidades cuyos autores publican en las revistas de comunicación, esta investigación muestra una diversidad mucho mayor, con más de cuarenta universidades españolas representadas en la muestra, y un cambio en las universidades de referencia. Mientras que la Universidad Complutense de Madrid, la Autónoma de Barcelona o la del País Vasco eran las más destacadas hace unos años (Míguez-González, 2011; Escribà-Sales; Cortiñas, 2013; Míguez-González; Baamonde-Silva; Corbacho-Valencia, 2014), hoy instituciones como las universidades Rey Juan Carlos, Rovira i Virgili o Málaga han tomado el relevo, con una presencia en la investigación que se ha ido incrementando en los últimos años (Gómez-Calderón; Roses, 2015).

Por otra parte, aunque muchas universidades pequeñas y periféricas, tanto públicas como privadas, consiguen tener presencia en la muestra por un único artículo publicado, se sigue manifestando la tendencia a la concentración de la investigación apuntada por Escribà-Sales y Cortiñas (2013).

Si se relaciona la institución con la autoría, se observa también que muchas de las universidades sólo cuentan con uno o dos autores que hayan publicado textos sobre comunicación organizacional en Scopus en el período analizado, mientras que unas pocas aportan un número de autores muy elevado y determinan, por tanto, el signo de la investigación en este tema.

De todos modos, se puede hablar de una importante variedad en la autoría, ya que el índice de transitoriedad es elevado (casi tres cuartas partes de los autores firman un único artículo de la muestra), un dato coherente con los estudios previos de Míguez-González (2011) y Míguez-González, Baamonde-Silva y Corbacho-Valencia (2014).
El profesional de la información es la revista española indexada en Scopus que más artículos sobre comunicación organizacional ha publicado en el último lustro 
En cuanto al sexo de los autores, se aprecia un ligero incremento en el porcentaje de autorías femeninas frente a masculinas en comparación con algunas investigaciones previas (Castillo-Esparcia; Carretón, 2010; Escribà-Sales; Cortiñas, 2013; Míguez-González; Baamonde-Silva; Corbacho-Valencia (2014) e incluso recientes (Goyanes; Rodríguez-Gómez; Rosique-Cedillo, 2018), aunque otros estudios específicos del ámbito de las relaciones públicas, el más próximo al objeto de estudio, ya señalan un mayor porcentaje de mujeres firmantes que de hombres (Míguez-González, 2011; Míguez-González; Corbacho-Valencia; Baamonde-Silva, 2016). La presente investigación detecta además que el porcentaje de mujeres que aparecen como primeras firmantes en los artículos colectivos es ligeramente superior a la proporción general de autoras y autores y que las tres autoras más productivas son mujeres.

Estas señales, si bien son discretas, pueden apuntar a un cierto incremento del peso femenino en la investigación de este campo, aunque para obtener conclusiones más válidas en este sentido sería necesario analizar estos datos en función del número de integrantes de las plantillas de investigadores de las universidades y del número de estudiantes de grados y masters del campo de la comunicación.

Por otra parte cabe destacar el elevado número de trabajos firmados por más de un autor, especialmente en 2017 y 2018. Tanto el porcentaje de trabajos colectivos, superior al 74\%, como el índice de cooperación superior a 2, suponen cambios significativos con respecto a estudios anteriores (Míguez-González, 2011; Escribà-Sales; Cortiñas, 2013; Míguez-González; Baamonde-Silva; Corbacho-Valencia, 2014; Costa-Sánchez, 2017), y determinan una reducción muy importante de los artículos de autoría individual o lo que es lo mismo, un incremento de las colaboraciones entre autores a la hora de investigar y publicar.

Esta tendencia alcista de los trabajos colectivos frente a los individuales se inició tímidamente hace una década (Fernández-Quijada, 2011) pero en los dos últimos años parece hacerse mucho más patente, al menos, en el campo de la comunicación organizacional. Fernández-Quijada (2011) busca la justificación a esta tendencia en el proceso de consolidación que han experimentado las ciencias de la comunicación con la emergencia de grupos y redes de investigación que complementan el trabajo de los tradicionales investigadores individuales. Se podría añadir que esta preferencia por el artículo colectivo en detrimento del individual probablemente también esté impulsada por los requisitos de publicación impuestos por Aneca y Cneai que, a pesar de penalizar en cierta medida los estudios firmados por un número de autores superior a la media en la especialidad para la consecución de acreditaciones y sexenios, impulsan a los investigadores a multiplicar y rentabilizar su trabajo a través de la colaboración con colegas académicos, como viene siendo tradicional en otros ámbitos y en las grandes revistas internacionales de comunicación, donde la coautoría es habitual (Méndez-Rátiva; Gregorio-Chaviano, 2014).

En cuanto a la temática, no se puede dejar de señalar la relevancia creciente del fenómeno de la comunicación online en revistas de cualquier origen geográfico, con un incremento de presencia de un $30 \%$ en relación con los 12 primeros años del siglo XXI (Míguez-González; Baamonde-Silva; Corbacho-Valencia, 2014). Otras tendencias que podrían apuntarse, comparando los datos con el estudio de Míguez-González; Baamonde-Silva; Corbacho-Valencia (2014), son la reducción del interés por la comunicación de crisis (de un 10,4\% a un 1,56\%), los estudios teóricos y de metainvestigación (de un $23 \%$ a un $7,81 \%$ ), la comunicación interna ( $8 \%$ a 3,13\%) o la gestión de eventos y el protocolo $(6,4 \%$ a 2,34\%), mientras que el interés por aspectos como el profesionalismo se mantiene $(9,8$ a 9,38), sobre todo en revistas internacionales, y el de las relaciones con los medios se incrementa (2,3\% a 5,47\%). Por lo que respecta a los sectores o tipo de organizaciones en las que se centra la investigación, se detecta una reducción del porcentaje de artículos de tipo general y un incremento de las investigaciones sobre empresas privadas de diversa índole.

Por lo que se refiere a la repercusión, el número de citas que reciben los artículos españoles sobre comunicación organizacional es bajo, especialmente en el caso de las revistas nacionales, aunque se mantiene en promedio dentro de los estándares habituales en las ciencias sociales en general y en la comunicación en particular (Méndez-Rátiva; Gregorio-Chaviano, 2014). Esto implica que mucha de la investigación generada no alcanza repercusión, lo que limita las posibilidades de que se establezca un debate académico enriquecedor en torno a su contenido. Asimismo la ausencia de correlación entre el número de citas y el número de autores firmantes pone de manifiesto que la autoría múltiple, aunque podría incrementar la red de investigadores vinculados con un trabajo y por tanto sus posibilidades de que sea citado, no implica necesariamente un incremento de la repercusión.

El presente estudio plantea en definitiva un panorama cuantitativamente estable en torno a la producción de investigación en comunicación organizacional pero dinámico en lo que se refiere a los autores y universidades que la investigan, con una elevada presencia femenina y una preferencia cada vez más marcada por los estudios colectivos que no se traduce en una mayor repercusión. Desde este punto de vista, el alcance de una mayor visibilidad y la consecuente ge- 
neración de debate académico en torno a una disciplina cuya definición resulta compleja y cuyas fronteras aún no están claramente delimitadas en el campo teórico, sería un objetivo muy deseable y fundamental de cara a su consolidación.

Una de las principales limitaciones de este estudio radica precisamente en la indefinición disciplinar, que ha dificultado la selección objetiva de la muestra, haciendo posible que algún artículo que debería estar aquí contemplado haya podido quedar fuera de los parámetros de búsqueda. La concreción de qué es la comunicación organizacional y qué elementos abarca continúa siendo por tanto un aspecto sobre el que cabe profundizar en futuras investigaciones. Por otra parte, el estudio, de carácter descriptivo, no analiza el enfoque o tratamiento concreto que se da en los artículos a las diferentes temáticas identificadas, aspecto que también podría ser de interés para una futura investigación.

\section{Referencias}

Almansa, Ana (2006). "Investigación sobre la formación de los directores de comunicación en las organizaciones". UNIrevista, v. 1, n. 3.

Andréu-Abela, Jaime (2001). "Las técnicas de análisis de contenido: una revisión actualizada”. Documento de trabajo S. http://www.albertomayol.cl/wp-content/uploads/2014/08/Andreu-Analisis-de-contenido.pdf

Arceo-Vacas, Alfredo (2005). "La imagen y las relaciones públicas versus la reputación y la comunicación corporativa". En: Otero-Alvarado, María-Teresa (coord.). I Congreso internacional de investigadores en relaciones públicas. Facultad de Comunicación de la Universidad de Sevilla, 17-18/09/2004. Sevilla: Asociación de Investigadores en Relaciones Públicas (AIRP), pp. 651-674. ISBN: 8460979970

https://idus.us.es/xmlui/handle/11441/38930

Baladrón-Pazos, Antonio J.; Correyero-Ruiz, Beatriz (2012). “Futuro de las revistas científicas de comunicación en España". El profesional de la información, v. 21, n. 1, pp. 34-42.

https://doi.org/10.3145/epi.2012.ene.05

Baladrón-Pazos, Antonio J.; Correyero-Ruiz, Beatriz; Manchado-Pérez, Benjamín (2014). "Tres décadas de investigación sobre publicidad en España. Análisis de las revistas científicas de comunicación (1980-2013)". Communication and society, v. 27, n. 4, pp. 49-71.

https://www.unav.edu/publicaciones/revistas/index.php/communication-and-society/article/view/35979 https://doi.org/10.15581/003.27.4.49-71

Bauer, Kathleen; Bakkalbasi, Nisa (2005). "An examination of citation counts in a new scholarly communication environment". D-lib magazine, v. 11, n. 9.

https://doi.org/10.1045/september2005-bauer

Benavides, Juan; Costa, Joan; Costa, Pere-Oriol; Fajula, Anna; Fürst, Philipp; Liria, Eduardo; Perdiguer, Agustín; Morales, Francisca; Ricarte, José-María; Roca, David; Rodríguez, Ángel; Soler, Pere; Ventura, Jordi (2001). Dirección de comunicación empresarial e institucional. Barcelona: Gestión 2000. ISBN: 8480884819

Borgman, Christine L.; Furner, Jonathan (2002). "Scholarly communication and bibliometrics". Annual review of information science and technology, v. 36, n. 1, pp. 2-72.

https://doi.org/10.1002/aris.1440360102

Castillo-Esparcia, Antonio (2005). Comunicación organizacional: teorías y estudios. Málaga: Clave Aynadamar. ISBN: 8480749504

Castillo-Esparcia, Antonio; Carretón, Carmen (2010). “Investigación en comunicación. Estudio bibliométrico de las revistas de comunicación en España”. Comunicación y sociedad, v. 23, n. 2, pp. 289-327.

https://www.unav.edu/publicaciones/revistas/index.php/communication-and-society/article/view/36234

https://doi.org/10.15581/003.23.2.289-327

Castillo-Esparcia, Antonio; Rubio-Moraga, Ángel; Almansa-Martínez, Ana (2012). "La investigación en comunicación. Análisis bibliométrico de las revistas de mayor impacto del ISI". Revista latina de comunicación social, n. 67. https://doi.org/10.4185/RLCS-067-955-248-270

Castillo-Esparcia, Antonio; Xifra, Jordi (2006). "Investigación bibliométrica de las tesis doctorales españolas sobre relaciones públicas (1965-2005)". Anàlisi: quaderns de comunicació i cultura, n. 34, pp. 141-161.

https://www.raco.cat/index.php/analisi/article/view/55449

Christensen, Lars-Thøger; Cornelissen, Josep (2011). "Bridging corporate and organizational communication: Review, development and a look to the future". Management communication quarterly, v. 25, n. 3, pp. 383-414.

https://doi.org/10.1177/0893318910390194 
Compte-Pujol, Marc; De-Urquijo, Beatriz; Matilla, Kathy (2016). "La investigación en marcas de territorio y diplomacia pública en España. Un estudio bibliométrico de las revistas científicas españolas especializadas en comunicación indexadas en Latindex (1980-2016)". Anales de documentación, v. 19, n.2.

https://doi.org/10.6018/analesdoc.19.2.255741

Cornelissen, Joep (2014). Corporate communication: A guide to theory and practice (4a ed.). London: Sage. ISBN: 9781 446274958

Costa, Joan (2011). El ADN del dircom: origen, necesidad, expansión y futuro de la dirección de comunicación. Tiana: Costa Punto Com. ISBN: 9788461502608

Costa-Sánchez, Carmen (2017). “Análisis de la productividad y visibilidad en Scopus de los investigadores españoles en comunicación". Observatorio (OBS*), v. 11, n. 3, pp. 1-16.

https://doi.org/10.15847/obsOBS11320171030

De-Castro, Adela (2015). Manual práctico de comunicación organizacional. Barranquilla: Editorial Universidad del Norte. ISBN: 8490743770

Delgado-López-Cózar, Emilio; Repiso, Rafael (2013). “El impacto de las revistas de comunicación: comparando Google Scholar Metrics, Web of Science y Scopus". Comunicar, v. 21, n. 41, pp. 45-52.

http://dx.doi.org/10.3916/C41-2013-04

Dilenschneider, Robert L. (2000). The corporate communications Bible. Everything you need to know to become a public relations expert. Beverly Hills, CA: New Millennium Press. ISBN: 9781893224087

Dircom (2013). Manual de la comunicación. Madrid: Dircom. ISBN: 9788461653614

Escribà-Sales, Eudald; Cortiñas, Sergi (2013). "La internacionalización y las coautorías en las principales revistas científicas de comunicación en España”. Comunicar, v. 21, n. 41, pp. 35-44.

https://doi.org/10.3916/C41-2013-03

Feeley, Thomas-Hugh (2008). "A bibliometric analysis of communication journals from 2002 to 2005". Human communication research, v. 34, n. 3, pp. 505-520.

https://doi.org/10.1111/j.1468-2958.2008.00330.x

Fernández-Quijada, David (2010). “El perfil de las revistas españolas de comunicación (2007-2008)”. Revista española de documentación científica, v. 33, n. 4, pp. 553-581.

https://doi.org/10.3989/redc.2010.4.756

Fernández-Quijada, David (2011). “De los investigadores a las redes: una aproximación tipológica a la autoría en las revistas españolas de comunicación". En: Actas del I Congreso nacional de metodología de la investigación en comunicación. Fuenlabrada: Asociación Española de Investigación de la Comunicación; Universidad Rey Juan Carlos. ISBN: 978 8469427132

http://eprints.rclis.org/18393/1/2011_aeic.pdf

García-Orosa, Berta (2006). “Aproximación teórica a la comunicación en el Tercer Sector. La necesaria reclasificación de la comunicación organizacional”. Telos: Cuadernos de comunicación e innovación, n. 69, pp. 51-59. https://telos.fundaciontelefonica.com/archivo/numero069

Goldhaber, Gerald M. (2003). Comunicación organizacional. México: Diana. ISBN: 9681316355 https://www.urbe.edu/UDWLibrary/InfoBook.do?id=8318

Gómez-Calderón, Bernardo; Roses, Sergio (2015). “Autores españoles altamente productivos en comunicación (20092013): perfil, impacto e internacionalización". Opción, v. 31, n. 4.

http://www.redalyc.org/pdf/310/31045569031.pdf

Goyanes, Manuel; Rodríguez-Gómez, Eduardo-Francisco; Rosique-Cedillo, Gloria (2018). “Investigación en comunicación en revistas científicas en España (2005-2015): de disquisiciones teóricas a investigación basada en evidencias". EI profesional de la información, v. 27, n. 6, pp. 1281-1291.

https://doi.org/10.3145/epi.2018.nov.11

Gutiérrez-García, Elena; Rodríguez-Salcedo, Natalia (2009). “50 años de relaciones públicas en España. De la propaganda y la publicidad a la gestión de la reputación”. Doxa comunicación, n. 9, pp. 13-33.

http://www.doxacomunicacion.es/es/hemeroteca/articulos?id=29

Guzmán, Vanessa (2012). Comunicación organizacional. México: Red Tercer Milenio. ISBN: 9786077330622 http://www.aliat.org.mx/BibliotecasDigitales/derecho_y_ciencias_sociales/Comunicacion_organizacional.pdf

Jablin, Frederic, M.; Putnam, Linda L. (2000). The new handbook of organizational communication. London: Sage. ISBN: 9780803955030 
Jones, Daniel E.; Baró, Jaume; Landa, Carmelo; Ontalba-Ruipérez, José-Antonio (2000). Investigación sobre comunicación en España. Aproximación bibliométrica a las tesis doctorales (1926-1998). Barcelona: ComCat.

León-Duarte, Gustavo A. (2006). "La comunicación organizacional en México. Enfoques, diseños y problemas en su desarrollo". Anàlisi: quaderns de comunicació i cultura, n. 34, pp. 287-304.

https://www.raco.cat/index.php/Analisi/article/view/55458

Losada-Díaz, José-Carlos (2004). Gestión de la comunicación en las organizaciones. Barcelona: Ariel. ISBN: 97884344 13030

Martínez-Nicolás, Manuel (2001). "Tendencias actuais da investigación sobre comunicación en Galizia”. Estudios de comunicación, n. 0, pp. 153-168.

https://www.researchgate.net/publication/260750196_Tendencias_actuais_da_investigacion_sobre_comunicacion_ en_Espana

Martínez-Nicolás, Manuel (2009). “La investigación sobre comunicación en España. Evolución histórica y retos actuales”. Revista latina de comunicación social, n. 64.

https://doi.org/10.4185/RLCS-64-2009-800-01-14

Martínez-Nicolás, Manuel; Saperas, Enric; Carrasco-Campos, Ángel (2017). "La investigación sobre periodismo en España. Análisis de los trabajos publicados en revistas científicas españolas en los últimos 25 años (1990-2014)”. Comunicación y sociedad, v. 30, n. 4, pp. 149-166.

https://www.unav.edu/publicaciones/revistas/index.php/communication-and-society/article/view/35764

https://doi.org/10.15581/003.30.4.149-166

Méndez-Rátiva, Claudia-Patricia; Gregorio-Chaviano, Orlando (2014). “Aproximación a la comunicación desde la perspectiva teórica y bibliométrica. Un análisis en Web of Science 2008-2012". Signo y pensamiento, v. 33, n. 64.

https://doi.org/10.11144/Javeriana.SyP33-64.actb

Míguez-González, María-Isabel (2011). "La investigación sobre relaciones públicas en las revistas españolas de comunicación (2008-2010)". En: Fernández-Souto, Ana-Belén; García-García, Francisco. VI Congreso internacional de investigación y relaciones públicas. Madrid: Icono 14, pp. 124-138. ISBN: 9788493807085

https://dialnet.unirioja.es/servlet/articulo?codigo $=6514237$

Míguez-González, María-Isabel (2015). “Algunas cuestiones terminológicas en torno a la comunicación corporativa”. En: Matilla, Kathy (ed.). Historia de la comunicación corporativa en Cataluña. Barcelona: UOC Ediciones, pp. 21-74. ISBN: 9788490648346

Míguez-González, María-Isabel; Baamonde-Silva, Xosé-Manuel; Corbacho-Valencia, Juan-Manuel (2014). “A bibliographic study of public relations in Spanish media and communication journals, 2000-2012". Public relations review, v. 40, n. 5 , pp. $818-828$.

https://doi.org/10.1016/j.pubrev.2014.08.002

Míguez-González, María-Isabel; Corbacho-Valencia, Juan-Manuel; Baamonde-Silva, Xosé-Manuel (2016). “Tendencias de investigación sobre relaciones públicas en revistas internacionales: el caso de Journal of public relations research 2012-2014". Revista internacional de relaciones públicas, v. 6, n. 12, pp. 5-24.

http://revistarelacionespublicas.uma.es/index.php/revrrpp/article/view/381

Morales, Francisca; Enrique, Ana-María (2007). "La figura del dircom. Su importancia en el modelo de comunicación integral". Anàlisi, n. 35, pp. 83-93.

https://www.raco.cat/index.php/Analisi/article/viewFile/74256/94425

Mut-Camacho, Magdalena (2006). “El director de comunicación: perfil de una nueva figura”. Fisec-Estrategias, v. 2, n. 5, pp. 3-23.

http://www.cienciared.com.ar/ra/usr/9/319/fisec_estrategias_m7_pp3_23.pdf

Noguera-Vivo, José-Manuel (2005). “Reseña de ‘Gestión de la comunicación en las organizaciones’ de José-Carlos Losada-Díaz (coord.)”. Sphera pública, n. 5, pp. 363-365.

http://www.redalyc.org/pdf/297/29700523.pdf

Pazmiño-Santacruz, Mauro-Roberto; González-Alonso, Jorge A. (2014). “Análisis exploratorio sobre las publicaciones relacionadas con la comunicación organizacional en pymes". Revista publicando, v. 1, n. 1, pp. 37-45.

https://www.rm/consultores.com/revista/index.php/crv/article/viewFile/5/pdf_5

Putnam, Linda L. (2002). "El cambio en las metáforas de la comunicación organizacional”. En: Putnam, Linda L.; Costa, Joan; Garrido, Francisco J. Comunicación empresarial: nuevas tendencias en comunicación para potenciar la estrategia empresarial. Barcelona: Gestión 2000, pp. 33-56. ISBN: 9788480887939 
Roca-Correa, David; Pueyo-Ayhan, Natalia (2012). "La productividad científica en comunicación a través de la revista Zer". Revista latina de comunicación social, n. 67.

https://doi.org/10.4185/RLCS-067-957-292-321

Saladrigas-Medina, Hilda (2005). "Comunicación organizacional: Matrices teóricas y enfoques comunicativos". Revista latina de comunicación social, v. 8, n. 60.

http://www.redalyc.org/articulo.oa?id $=81986008$

Soriano-Clemente, Jaume (2008). "El efecto Aneca". I+C Investigar a comunicación. Actas y memoria final. Congreso internacional fundacional AE-IC, pp. 1-18. Santiago de Compostela. ISBN: 9788461238163

Timoteo-Álvarez, Jesús (2013). Manejo de la comunicación organizacional: Espacios, herramientas y tendencias en gestión de negocios. Ediciones Díaz de Santos. ISBN: 9788499694474

Torres-Alonso, María-del-Carmen; Alejo-Becerra, José-Alberto (2011). "Surgimiento y desarrollo de la comunicación organizacional y su relación con otras ciencias". Contribuciones a las ciencias sociales, julio 2011.

http://www.eumed.net/rev/cccss/13/taab.htm

Valle, Mónica (2003). “La comunicación organizacional de cara al siglo XXI”. Razón y palabra, n. 32. http://www.razonypalabra.org.mx/anteriores/n32/mvalle.html

Van-Riel, Cees M. (1997). Comunicación corporativa. Madrid: Prentice Hall. ISBN: 9788489660076

Van-Riel, Cees B. (2003). “Nuevas formas de la comunicación organizacional”. Razón y palabra, n. 34. http://www.razonypalabra.org.mx/anteriores/n34/cvanriel.html

Van-Riel, Cees B.; Fombrun, Charles J. (2007). Essentials of corporate communication: Implementing practices for effective reputation management. London: Routledge. ISBN: 9780415328265

Vicente-Torrico, David (2017). “Estudio bibliométrico de la producción científica sobre narrativa transmedia en España hasta 2016: Análisis descriptivo de las 20 principales revistas de comunicación españolas según Google Scholar Metrics (h5)". AdComunica, n. 14, pp. 141-160.

http://www.adcomunicarevista.com/ojs/index.php/adcomunica/article/view/406

Villafañe, Justo (1993). Imagen positiva: gestión estratégica de la imagen de las empresas. Madrid: Pirámide. ISBN: 978 8436807868

Wilcox, Dennis L.; Cameron, Glen T.; Xifra, Jordi (2012). Relaciones públicas: estrategias y tácticas. Madrid: Pearson. ISBN: 9788483228135

Xifra, Jordi (2005). Planificación estratégica de relaciones públicas. Barcelona: Paidós. ISBN: 9788449317781

Xifra, Jordi; Castillo-Esparcia, Antonio (2006). "Forty years of doctoral public relations research in Spain: A quantitative study of dissertation contribution to theory development". Public relations review, v. 32, n. 3, pp. 302-308.

https://doi.org/10.1016/j.pubrev.2006.05.004

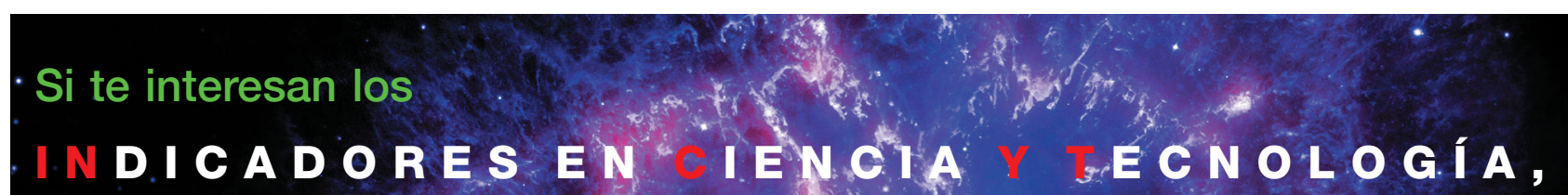

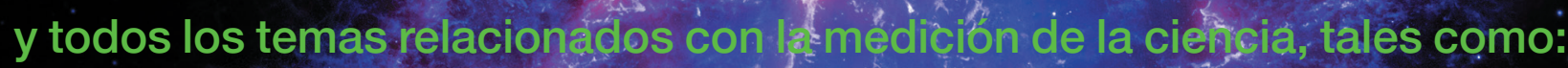

Análisis de citás, Normalización dén nombreš e instituciones: Impacto de

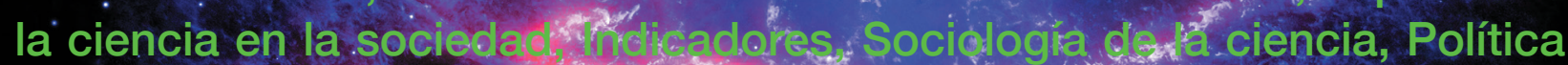

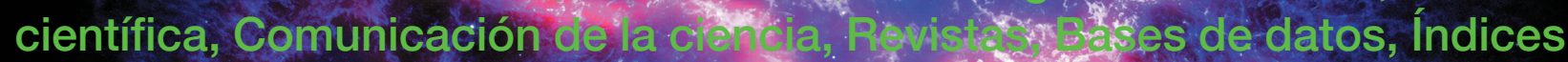

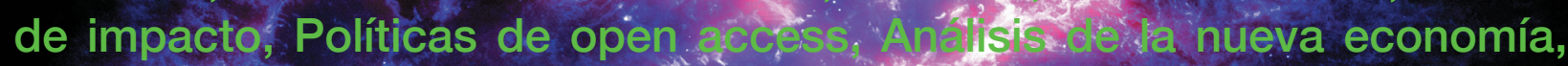
Mujer y ciencia, etc.

\section{Entonces}

http://wWw.rediris.es/list/info/incyt.htmI 